\title{
Noncommunicable Diseases Household Survey Data Analysis, Sana'a City, Yemen, 2017
}

Ghamdan Gamal Alkholidy ${ }^{1}$, MBBS; Labiba Saeed Anam ${ }^{1}$, MD; Abdulwahed Al Serouri ${ }^{1}$, PhD; Chaoyang Li $^{2}, \mathrm{MD}_{\text {, }}$ $\mathrm{PhD}$

\author{
${ }^{1}$ Yemen Field Epidemiology Training Program, Ministry of Public Health and Population, Sana'a, Yemen \\ ${ }^{2}$ Division of Global Health Protection, Center for Global Health, Centers for Disease Control and Prevention, Atlanta, GA, United States
}

\section{Corresponding Author:}

Ghamdan Gamal Alkholidy, MBBS

Yemen Field Epidemiology Training Program

Ministry of Public Health and Population

Alsteen St, Bldg 23

Sana'a, 000

Yemen

Phone: 967771530764

Email: ghamdangamal@gmail.com

\section{Abstract}

Background: Noncommunicable diseases (NCDs) kill 41 million people each year, accounting for $71 \%$ of all deaths globally. The burden of NCDs is rising faster in developing countries of the Middle East than in other regions. The morbidity and mortality of NCDs are still not well-studied in Yemen.

Objective: The aim of this study was to describe the epidemiology of NCDs in Sana'a City, Yemen, for 2017.

Methods: Raw data of a house-to-house survey that was conducted by the Ministry of Public Health and Population in 2017 were analyzed. Data were collected from household heads who were asked if any household member had one of the following five NCDs: hypertension (HTN), diabetes (DM), bronchial asthma (BA), mental disorders (MD), and epilepsy. Data were entered and analyzed using Epi info 7.2. For calculations of prevalence, 2017 projections from the 2004 census were used.

Results: A total of 241,310 households were surveyed (1,592,646 household members), 59,061 (24.48\%) of which included 70,178 members who had at least one NCD. The overall prevalence of NCDs was $4.4 \%$. The disease-specific prevalence was as follows: HTN, 2.3\%; DM, 2.2\%; BA, 0.4\%; MD, 0.27; and epilepsy, 0.19\%. The overall NCD prevalence was significantly higher among females than males $(5.1 \%$ vs $3.8 \%$; odds ratio [OR] 1.35, 95\% CI 1.33-1.35), which was also the case for the prevalence of HTN (3.1\% vs 1.6\%; OR 1.94, 95\% CI 1.90-1.98), DM (2.3\% vs $2.1 \%$; OR $1.11,95 \%$ CI $1.09-1.13)$, and BA (0.5\% vs $0.3 \%$; OR $1.56,95 \%$ CI 1.49-1.65). In contrast, the prevalence of MD was significantly higher among males than females $(0.35 \%$ vs $0.16 \%$; OR 2.2, 95\% CI 2.06-2.31). The prevalence of NCDs progressively increased with age. Nearly $18 \%$ of patients had more than one NCD; $35.2 \%$ of the patients with HTN also had DM.

Conclusions: One-quarter of the surveyed households had at least one member with one or more of the five NCDs and the overall prevalence of NCDs was $4.4 \%$. These data reflect only the tip of the iceberg as the findings are based on self-reported diagnosed cases rather than standardized measures. More attention to NCDs, strengthened health care provision, the ability to obtain high-reliability data, an NCDs stepwise survey, and establishing an NCDs surveillance system are recommended.

(iproc 2022;8(1):e36566) doi: $10.2196 / 36566$

\section{KEYWORDS}

NCDs; survey; hypertension; diabetes; bronchial asthma; mental disorder; epilepsy; Yemen 
Edited by Y Khader; this is a non-peer-reviewed article. Submitted 18.01.22; accepted 19.01.22; published 07.02.22.

Please cite as:

Alkholidy GG, Anam LS, Al Serouri A, Li C

Noncommunicable Diseases Household Survey Data Analysis, Sana'a City, Yemen, 2017

iproc 2022;8(1):e36566

URL: https://www.iproc.org/2022/1/e36566

doi: $\underline{10.2196 / 36566}$

PMID:

(C) Ghamdan Gamal Alkholidy, Labiba Saeed Anam, Abdulwahed Al Serouri, Chaoyang Li. Originally published in Iproceedings (https://www.iproc.org), 07.02.2022. This is an open-access article distributed under the terms of the Creative Commons Attribution License (https://creativecommons.org/licenses/by/4.0/), which permits unrestricted use, distribution, and reproduction in any medium, provided the original work, first published in Iproceedings, is properly cited. The complete bibliographic information, a link to the original publication on https://www.iproc.org/, as well as this copyright and license information must be included. 